MS27 Dynamical refinement of electron diffraction data

\author{
Chairs: Mauro Gemmi, Richard Beanland
}

\section{MS27-P1 Looking for the Potential in Digital Large-Angle Electron Diffraction Patterns}

Richard Beanland ${ }^{1}$

\section{University of Warwick}

email: r.beanland@warwick.ac.uk

The sensitivity of convergent beam electron diffraction (CBED) to a crystal's structure and electron density has been known for a long time. The technique has the advantage of sampling material limited only by the size of the electron beam and the thickness of the specimen, i.e. a volume that can easily be less than 5000 cubic nanometres. However, the small electron wavelength of high energy electrons leads to very small Bragg angles, which severely restricts the convergence angle that can be used. The problem becomes worse for materials with larger lattice parameters, often with the result that insufficient information is obtained from CBED to do anything useful.

Computer control of transmission electron microscopes and digital image capture allows hundreds, or thousands, of CBED patterns to be collected automatically. The patterns can then be combined to construct a single 'digital' large-angle convergent beam electron diffraction (D-LACBED) pattern that has no restrictions due to Bragg angle. These patterns have a wealth of detail and, for example, symmetry determination becomes straightforward. Examples are given from materials that would be difficult or impossible using conventional CBED.

While analysis of the intensities in CBED patterns has been shown to yield information about crystal potentials even providing sufficient detail to show the redistribution of electrons between atoms (i.e. bonding effects) - there has been less work on LACBED patterns. Careful experimentation and energy filtered imaging is needed for CBED work, since reliable answers usually require elastically-scattered intensities to be measured with accuracies better than $1 \%$. For D-LACBED patterns, it may be hoped that the large amount of data contains some regions that are much more sensitive than others. Thus, the sensitivity of D-LACBED patterns to changes in the crystal potential is investigated using Bloch wave simulations. It is found that dynamical diffraction effects often produce large changes in the intensity of multiple diffracted beams from the variation of a single Fourier coefficient that describes the crystal potential. It thus seems possible that highly accurate measurement of crystal structure and bonding on a routine basis is readily achievable with a modern computer controlled TEM without the need for energy filtering.
Experiment

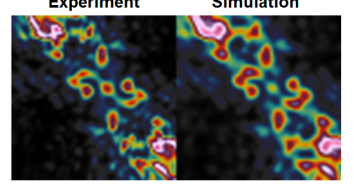

Difference

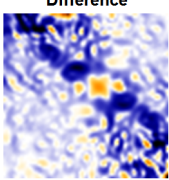

Figure 1. Experimental and simulated 111 D-LACBED pattern from the [110] zone axis of GaAs. The simulated pattern uses Kirkland scattering factors and is optimised for specimen thickness, Debye-Waller factor and absorption. Large differences in intensity remain $(>30 \%)$, presumably due to bonding effects.

Keywords: D-LACBED, dynamical electron diffraction, refinement

ynamical

\section{diffraction,}

